\title{
ON SPECTRAL THEORY AND CONVEXITY
}

\author{
BY
}

C. K. FONG AND LOUISA LAM ${ }^{1}$

\begin{abstract}
A compact convex set $K$ in a locally convex algebra is said to be a spectral carrier if, for all $x, y \in K$, we have $x y=y x \in K$ and $x+y-x y \in K$. We show that if a compact convex set $K$ is a spectral carrier, then the idempotents in $K$ are exactly the extreme points of $K$ and form a complete lattice. Conversely, if a compact set $K$ is a closed convex hull of a lattice of commuting idempotents, then $K$ is a spectral carrier. Furthermore, a metrizable spectral carrier is a Choquet simplex if and only if its extreme points form a chain of idempotents.
\end{abstract}

1. Introduction. The purpose of the present paper is to describe a general spectral theory for certain elements in a locally convex algebra over $\mathbf{C}$ or $\mathbf{R}$ from Choquet theory's point of view.

By a locally convex algebra we mean an algebra having an identity and a locally convex topology for which the multiplication is separately continuous. In $\$ 2$, we will consider elements in a locally convex algebra $A$ which are contained in some compact convex set $K$ whose extreme points form a lattice of commuting idempotents. In $\S 3$, we show that such $K$ is a simplex if and only if its extreme points form a chain of idempotents. If $h$ is a hermitian operator on a Hilbert space $H$ satisfying $0 \leqslant h \leqslant 1$ with $h=\int_{0}^{1} \lambda d e_{\lambda}$ as its spectral decomposition, we can show, by means of integration by parts, that $h$ can be expressed as $\int_{c} e d \mu(e)$, where $C$ is the weak closure of $\left\{e_{\lambda}: 0 \leqslant \lambda \leqslant 1\right\}$ and $\mu$ is a probability measure on $C$; thus $h$ is contained in the simplex $\overline{\operatorname{co}}(C)$, the weak closure of the convex hull of $C$, whose extreme points form a chain of projections.

In case that the algebra $A$ is finite-dimensional, the situation is much simpler, as shown in the following proposition.

Proposition 1.1. Let $A$ be a finite-dimensional algebra (over $\mathbf{C}$ or $\mathbf{R}$, with identity 1) and let $x$ be in $A$. Then the following conditions are equivalent.

(a) $x$ can be expressed as $\sum_{j=1}^{m} \mu_{j} f_{j}$, where $\mu_{j}$ are real numbers satisfying $0 \leqslant \mu_{j}<1$ and $f_{j}$ are idempotents in $A$ such that $f_{i} f_{j}=0$ if $i \neq j$.

(b) $x$ can be expressed as $\sum_{k=1}^{n} \lambda_{k} e_{k}$ where $\lambda_{k}>0, \sum_{k=1}^{n} \lambda_{k}=1$ and $e_{k}$ are idempotents satisfying $e_{k} e_{j}=e_{j} e_{k}=e_{k}$ if $k \geqslant j$.

(c) $x$ can be expressed as a convex combination of commuting idempotents in $A$.

Received by the editors August 25, 1979.

AMS (MOS) subject classifications (1970). Primary 47D20; Secondary 46H99, 47B15.

Key words and phrases. Extreme point, spectral carrier, spectral decomposition, facial ideal, chain of idempotents, simplex.

'The contribution of the second author represents part of her thesis under the supervision of Professor L. T. Gardner. 
(d) There is a compact convex set $K$ containing $x$ with the following property: if $y$, $z \in K$, then $y z=z y \in K$ and $y+z-y z \in K$.

Proof. (a) $\Rightarrow(b)$. Without the loss of generality, we may assume that

$$
x=\sum_{j=1}^{m} \mu_{j} f_{j} \text { with } 0 \leqslant \mu_{1}<\mu_{2}<\cdots<\mu_{m}<1 .
$$

Let $\lambda_{1}=\mu_{1}$ and $\lambda_{j}=\mu_{j}-\mu_{j-1}$ for $j=2,3, \ldots, m$. Then $\lambda_{k} \geqslant 0$ for all $k$ and

$$
\sum_{k=1}^{m} \lambda_{k}=\mu_{m}<1
$$

Now we have

$$
x=\sum_{j=1}^{m}\left(\sum_{k=1}^{j} \lambda_{k}\right) f_{j}=\sum_{k=1}^{m} \lambda_{k} e_{k},
$$

where $e_{k}=\sum_{j=k}^{m} f_{j}$. Then $e_{k} e_{k+1}=e_{k+1} e_{k}=e_{k+1}$ for $k=1,2, \ldots, m-1$. In case $\mu_{m}=1$, we are done. Otherwise we let $e_{m+1}=0$ and $\lambda_{m+1}=1-\mu_{m}$. Then $x=\sum_{k=1}^{m+1} \lambda_{k} e_{k}$, where $\lambda_{k} \geqslant 0$ and $\sum_{k=1}^{m+1} \lambda_{k}=1$.

(b) $\Rightarrow$ (c). Obvious.

(a) $\Rightarrow(d)$. Let $K$ be the set of those elements in $A$ which can be expressed as $\sum_{j=1}^{m} \nu_{j} f_{j}$ with $0<\nu_{j}<1$. Then $x \in K$ and $K$ has the required property.

(d) $\Rightarrow$ (c). Since $x$ can be expressed as a convex combination of extreme points of $K$, it suffices'to show that each extreme point $e$ is an idempotent. By assumption, both $e^{2}$ and $2 e-e^{2}$ are in $K$. Since $e$ is an extreme point, from the identity

$$
e=\frac{1}{2} e^{2}+\frac{1}{2}\left(2 e-e^{2}\right)
$$

we obtain $e^{2}=e$.

(c) $\Rightarrow$ (a). Suppose that $x=\sum_{k=1}^{m} \lambda_{k} e_{k}$ with $\lambda_{k}>0, \sum_{k=1}^{m} \lambda_{k}=1$ and $e_{1}, \ldots, e_{m}$ are commuting idempotents. We show (a) by induction on $m$. Assume that (a) holds if $m=s$. Now we consider the case $m=s+1$. If $\lambda_{s+1}=0$, then (a) follows from the induction hypothesis. Hence we assume $\lambda_{s+1} \neq 0$. Let

$$
x_{1}=\sum_{k=1}^{s}\left(1-\lambda_{s+1}\right)^{-1} \lambda_{k} e_{k} .
$$

Since $x_{1}$ is a convex combination of $e_{1}, \ldots, e_{s}$, by the induction hypothesis, $x_{1}=\sum_{k=1}^{r} \mu_{k} f_{k}$ for some reals $\mu_{k}$ with $0<\mu_{k}<1$ and some idempotents $f_{k}$ with $f_{j} f_{k}=0$ if $j \neq k$. We may assume $\sum_{k=1}^{r} f_{k}=1$. Now

$$
\begin{aligned}
x & =\left(1-\lambda_{s+1}\right) x_{1}+\lambda_{s+1} e_{s+1}=\sum_{k=1}^{r}\left(1-\lambda_{s+1}\right) \mu_{k} f_{k}+\lambda_{s+1} e_{s+1} \\
& =\sum_{k=1}^{r}\left(\left(1-\lambda_{s+1}\right) \mu_{k}+\lambda_{s+1}\right) f_{k} e_{s+1}+\sum_{k=1}^{r}\left(\left(1-\lambda_{s+1}\right) \mu_{k}\right) f_{k}\left(1-e_{s+1}\right)
\end{aligned}
$$

with $0 \leqslant\left(1-\lambda_{s+1}\right) \mu_{k} \leqslant\left(\left(1-\lambda_{s+1}\right) \mu_{k}+\lambda_{s+1}\right)<1$. Hence the statement (a) is true for $m=s+1$. 
Next we consider the uniqueness of the expression of $x$ in (b) of the above proposition. Suppose that

$$
x=\sum_{k=1}^{n} \lambda_{k} e_{k}=\sum_{k=1}^{n} \tilde{\lambda}_{k} e_{k}
$$

where $e_{k} e_{j}=e_{j} e_{k}=e_{k}$ and $e_{k} \neq e_{j}$ if $k>j$. Then we have

$$
\left(1-e_{2}\right) x=\lambda_{1} e_{1}\left(1-e_{2}\right)=\tilde{\lambda}_{1} e_{1}\left(1-e_{2}\right) \text {. }
$$

Since $e_{1} \neq e_{2}$, we have $e_{1}\left(1-e_{2}\right) \neq 0$ and hence $\lambda_{1}=\tilde{\lambda}_{1}$. By induction, we have $\lambda_{k}=\tilde{\lambda}_{k}$ for all $k$. This proves the following:

Proposition 1.2. The convex hull of idempotents $e_{1}, e_{2}, \ldots, e_{n}$ with the property $e_{k} e_{k+1}=e_{k+1} e_{k}=e_{k+1}$ for $k=1, \ldots, n-1$ is a simplex.

2. Spectral carriers. The following definition is motivated by condition (d) in Proposition 1.1.

Definition 2.1. A compact convex set $K$ in a locally convex algebra $A$ is called a spectral carrier if it satisfies the followng three conditions:

(a) $x, y \in K$ implies $x y=y x$,

(b) $x, y \in K$ implies $x y \in K$,

(c) $x, y \in K$ implies $x+y-x y \in K$.

Condition (b) of the above definition says that $K$ is closed under multiplication. If 1 is the identity of $A$, then condition (c) says that $1-K=\{1-x: x \in K\}$ is closed under multiplication.

REMARK. The term "spectral carrier" suggests that elements in $K$ have certain spectral properties. To illustrate this point, next we show an analogue of the fact that if $p$ is a positive operator on a Hilbert space $H, \xi \in H$ and $p^{2} \xi=0$, then $p \xi=0$.

Proposition 2.1. If $K$ is a spectral carrier in a locally convex algebra $A, x \in K$, $y \in A$ and $x^{2} y=0$, then $x y=0$. (Note that we do not assume $x$ and $y$ commute.)

Proof. From $x \in K$ and condition (c) in Definition 2.1, we have $x_{1}=2 x-x^{2}$ $\in K$. Since $x^{2} y=0$, we have $x_{1} y=2 x y$. Now $x_{1}^{2} y=x_{1}\left(x_{1} y\right)=x_{1}(2 x y)=$ $2 x\left(x_{1} y\right)=2 x(2 x y)=4 x^{2} y=0$. On the other hand, since $x_{1} \in K$, we have $2 x_{1}-$ $x_{1}^{2} \in K$. Hence $2 x_{1} y=2 x_{1} y-x_{1}^{2} y \in K y$, or $4 x y \in K y$. By induction, we can show that $2^{n} x y \in K y$ for all $n \geqslant 1$. Since $K y$ is compact, we must have $x y=0$.

The argument used for proving $(d) \Rightarrow(c)$ in Proposition 1.1 gives the following result:

Proposition 2.2. Extreme points of a spectral carrier are idempotents.

The main result of the present section is that, conversely, idempotents in a spectral carrier are extreme points and they form a complete lattice. The idea of the proof is to establish a one-one correspondence between extreme points of $K$ and certain faces of $K$. In what follows, $K$ always stands for a spectral carrier in a locally convex algebra and $\partial_{e} K$ stands for the set of all extreme points of $K$. Recall 
that a subset $F$ of $K$ is called a face of $K$ if $F$ is convex and extremal, that is, for $x$, $y \in K,(x+y) / 2 \in F$ if and only if $x, y \in F$.

Proposition 2.3. If $F$ is a face of $K$, then $x, y \in F$ implies $x y \in F$ and $x+y-x y \in F$. Hence closed faces of $K$ are spectral carriers.

Proof. Let $x, y$ be in $F$. Since $F$ is convex, we have $(x+y) / 2 \in F$. From $x$, $y \in K$ we have $x y \in K$ and $x+y-x y \in K$. From the trivial identity

$$
\frac{1}{2}(x y+(x+y-x y))=\frac{1}{2}(x+y) \in F
$$

and the extremal property of $F$ it follows that both $x y$ and $x+y-x y$ are in $F$.

Since $e \in \partial_{e} K$ if and only if the singleton $\{e\}$ is a face of $K$, Proposition 2.2 is also a consequence of Proposition 2.3.

Definition 2.2. A nonempty subset $F$ of $K$ is called a facial ideal of $K$ if $F$ is a closed face with the property $F K \subseteq F$, that is, if $x \in F$ and $y \in K$, then $x y \in F$.

Proposition 2.4. If $e \in \partial_{e} K$, then $F=e K$ is a facial ideal.

Proof. It is easy to see that $F$ is compact, convex and $F K \subseteq F$. It remains to show that $F$ is extremal in $K$. Suppose that $x, y \in K$ and $z=(x+y) / 2 \in F$. By Proposition 2.2, we have $e^{2}=e$. From $z \in e K$ and $e^{2}=e$ it is easy to see that $e z=z$, that is

$$
(e x+e y) / 2=(x+y) / 2
$$

or

$$
e=((e+x-e x)+(e+y-e y)) / 2 \text {. }
$$

Since $e+x-e x$ and $e+y-e y$ are in $K$ and $e \in \partial_{e} K$, we have

$$
e=e+x-e x=e+y-e y .
$$

Hence $x=e x$ and $y=e y$. In other words, $x, y \in F$.

Next we show that $K$ has a smallest idempotent.

Proposition 2.5. There exists an idempotent $e_{0}$ in $\partial_{e} K$ such that $e_{0} x=e_{0}$ for all $x \in K$.

Proof. Consider the family

$$
\mathscr{F}=\left\{e K: e \in \partial_{e} K\right\}
$$

By Proposition 2.4, $\mathscr{F}$ is a family of facial ideals. Hence the intersection $K_{0}=\cap \mathscr{F}$ is also a facial ideal, provided that it is nonempty.

Note that if $e_{1}, \ldots, e_{n}$ are in $\partial_{e} K$, then

$$
\left(e_{1} \cdots e_{n}\right) K \subseteq e_{1} K \cap \cdots \cap e_{n} K .
$$

Hence $\mathscr{F}$ has the finite intersection property. The compactness of $K$ guarantees that $K_{0}=\cap \mathscr{F}$ is nonempty.

By Proposition 2.3, $K_{0}$ is a spectral carrier. Let $e_{0} \in \partial_{e} K_{0}$. Since $K_{0}$ is a face of $K$, we have $\partial_{e} K_{0} \subseteq \partial_{e} K$. Therefore $e_{0} \in \partial_{e} K$. 
Finally we show that $e_{0} x=e_{0}$. If $e \in \partial_{e} K$, then $e_{0} \in e K$ and hence $e_{0} e=e_{0}$. If $x$ is a convex combination of extreme points, say, $x=\sum_{k=1}^{n} \lambda_{k} e_{k}$ with $e_{k} \in \partial_{e} K$, $\lambda_{k} \geqslant 0$ and $\sum_{k=1}^{n} \lambda_{k}=1$, then

$$
e_{0} x=\sum_{k=1}^{n} \lambda_{k} e_{0} e_{k}=\sum_{k=1}^{n} \lambda_{k} e_{0}=e_{0}
$$

In general, if $x \in K$, then, by Krein-Mil'man's Theorem, there exists a net $\left\{x_{\alpha}\right\}$ of convex combinations of $\partial_{e} K$ converging to $x$ and hence we obtain

$$
e_{0} x=e_{0} \lim x_{\alpha}=\lim e_{0} x_{\alpha}=e_{0} \text {. }
$$

Corollary 2.6. If $0 \in K$, then $0 \in \partial_{e} K$.

Corollary 2.7. There exists an idempotent $e_{1}$ in $\partial_{e} K$ such that $e_{1} x=x$ for all $x \in K$.

Proof. Note that $1-K=\{1-x: x \in K\}$ is also a spectral carrier. Apply Proposition 2.5 to $1-K$, we can find an extreme point $e$ of $1-K$ such that $e(1-x)=e$ for all $x \in K$. Let $e_{1}=1-e$. Then $e_{1} \in \partial_{e} K$ and $e_{1} x=x$ for all $x$ in $K$.

It follows from the proof of Proposition 2.5 that

COROllary 2.8. The intersection of an arbitrary family of facial ideals is a facial ideal.

The next result is the converse of Proposition 2.4.

Proposition 2.9. If $F$ is a facial ideal of $K$, then there is an extreme point e of $K$ such that $F=e K$.

Proof. Apply Corollary 2.7 to the spectral carrier $F$, we obtain an idempotent $e$ in $\partial_{e} F$ such that $F=e F$. From the fact that $F$ is a face of $K$, we have $\partial_{e} F \subseteq \partial_{e} K$ and hence $e \in \partial_{e} K$. Since $e \in F$, we have $e K \subseteq F k \subseteq F$. On the other hand, since $F \subseteq K$, we have $F=e F \subseteq e K$. Therefore $F=e K$.

THEOREM 2.10. An element in a spectral carrier $K$ is an idempotent if and only if it is an extreme point of $K$.

Proof. The "if" part, which is the easier part, is just Proposition 2.2. To show the "only if" part, suppose that $e \in K$ and $e^{2}=e$. We claim that $F=e K$ is a facial ideal. It is clear that $F$ is compact, convex and $F K \subseteq F$. Suppose that $x, y \in K$ and $z=(x+y) / 2$ is in $F$. We have to show that $x, y$ are also in $F$. Since $e \in K$, we have $0=e(1-e) \in(1-e) K$. It is easy to check that $(1-e) K$ is a spectral carrier. Hence, by Corollary $2.6,0$ is an extreme point of $(1-e) K$. On the other hand, from $z=(x+y) / 2 \in e K$ we have $e z=z$ or

$$
((1-e) x+(1-e) y) / 2=0 .
$$

It follows that $(1-e) x=(1-e) y=0$ or $x=e x$ and $y=e y$. Thus we have shown $F$ is a face and hence a facial ideal of $K$. By Proposition 2.7, there exists an extreme point $e_{1}$ of $K$ such that $F=e_{1} K$. From the fact that both $e_{1}$ and $e$ are idempotents and $e K=e_{1} K$ it is easy to see that $e=e_{1}$. Therefore $e \in \partial_{e} K$. 
REMARK. It follows from the above theorem that if $K_{1}$ and $K_{2}$ are spectral carriers, then $K_{1} \subseteq K_{2}$ if and only if $\partial_{e} K_{1} \subseteq \partial_{e} K_{2}$. Also, if $\left\{K_{\alpha}\right\}$ is a family of spectral carriers and its intersection $K=\bigcap_{\alpha} K_{\alpha}$ is nonempty, then $K$ is also a spectral carrier and

$$
\partial_{e} K=\bigcap_{\alpha} \partial_{e} K_{\alpha}
$$

We define an ordering among idempotents in an algebra as follows. For idempotents $e_{1}, e_{2}$ in $A$, we put $e_{1} \leqslant e_{2}$ if $e_{1} e_{2}=e_{2} e_{1}=e_{1}$. With this ordering, it is easy to check that if $e_{1}, e_{2}$ are commuting idempotents in $A$, then $e_{1} \wedge e_{2}$ (the infimum of $\left.\left\{e_{1}, e_{2}\right\}\right)$ and $e_{1} \vee e_{2}$ exist, in fact,

$$
e_{1} \wedge e_{2}=e_{1} e_{2}, \quad e_{1} \vee e_{2}=e_{1}+e_{2}-e_{1} e_{2}
$$

Thus the idempotents in $K$ form a lattice. Let $\mathscr{F}$ be the family of all facial ideals of $K$. Then the mapping $\Phi: \partial_{e} K \rightarrow \mathcal{F}$ given by $\Phi(e)=e K$ is a one-one correspondence between the idempotents and the facial ideals of $K$. Obviously $e_{1}<e_{2}$ if and only if $\Phi\left(e_{1}\right) \subseteq \Phi\left(e_{2}\right)$. Also

$$
\Phi\left(e_{1} \wedge e_{2}\right)=\Phi\left(e_{1}\right) \cap \Phi\left(e_{2}\right) .
$$

If $\left\{e_{\alpha}\right\}$ is a family of idempotents in $K$, then, by Corollary 2.8 , the intersection $\cap_{\alpha} \Phi\left(e_{\alpha}\right)$ is a facial ideal and hence equals $\Phi(e)$ for some $e \in \partial_{e} K$. It is easy to see that $e$ is the infimum of $\left\{e_{\alpha}\right\}$ and thus

$$
\Phi\left(\bigwedge_{\alpha} e_{\alpha}\right)=\bigcap_{\alpha} \Phi\left(e_{\alpha}\right) \text {. }
$$

This proves part (a) and (b) of the following theorem.

THEOREM 2.11. Let $K$ be a spectral carrier. Then the following statements hold.

(a) The extreme boundary $\partial_{e} K$ forms a complete lattice.

(b) If $\left\{e_{\alpha}\right\}$ is a subset of $\partial_{e} K$ with $e$ as its infimum, then $e K=\cap_{\alpha} e_{\alpha} K$.

(c) If $\left\{e_{\alpha}\right\}$ is a decreasing (or increasing) net of idempotents in $K$, then $\lim e_{\alpha}=e$ exists and $e \in \partial_{e} K$.

Proof of (c). Let $e$ be the infimum of the decreasing net $\left\{e_{\alpha}\right\}$. Suppose for this moment that $\lim e_{\alpha}=x$ does exist. For $\beta \geqslant \alpha$, we have $e_{\alpha} e_{\beta}=e_{\beta}$. Hence, when $\alpha$ is fixed, we have

$$
x=\lim _{\beta} e_{\beta}=\lim _{\beta} e_{\alpha} e_{\beta}=e_{\alpha} \lim _{\beta} e_{\beta}=e_{\alpha} x .
$$

Therefore $x \in e_{\alpha} K$ for all $\alpha$. By (b), we have $x \in e K$ from which it follows that $x e=x$. On the other hand, since $e \in e_{\alpha} K$ for all $\alpha$, we have $e e_{\alpha}=e$. Hence

$$
e=\lim _{\alpha} e e_{\alpha}=e \lim _{\alpha} e_{\alpha}=e x .
$$

Therefore $e=x$. The same argument shows that if a subnet of $\left\{e_{\alpha}\right\}$ is convergent, then it must converge to $e$. By the compactness of $K$, it follows that $\lim e_{\alpha}=e$.

RemarK. In general, $\partial_{e} K$ is not a closed set in $K$. For example, let $A=L^{\infty}[0,1]$ with the weak*-topology. Then $K=\left\{x \in L^{\infty}[0,1]: 0<x<1\right\}$ is a spectral carrier and $\partial_{e} K$ is the set of all indicator functions, which is not closed under the weak*-topology. 
COROLlaRY 2.11'. If $K$ is a spectral carrier satisfying $K=1-K$ then $\partial_{e} K$ is a complete Boolean algebra of idempotents.

Next we show that, under a suitable condition, the closed convex hull of a lattice of idempotents is a spectral carrier.

Proposition 2.12. If $E$ is a set of commuting idempotents in $A$ such that, for all $e_{1}$, $e_{2} \in E$, both $e_{1} \wedge e_{2} \equiv e_{1} e_{2}$ and $e_{1} \vee e_{2} \equiv e_{1}+e_{2}-e_{1} e_{2}$ are in $E$ and the closed convex hull $J=\overline{c o}(E)$ is compact, then $J$ is a spectral carrier.

Proof. We write $\operatorname{co}(E)$ for the convex hull of $E$. Let $x, y \in \operatorname{co}(E)$. Then $x, y$ can be expressed as $\Sigma \lambda_{k} e_{k}$ and $\Sigma \mu_{j} f_{j}$ respectively, where $e_{k}, f_{j} \in E, \lambda_{k}>0, \mu_{j}>0$ and $\sum \lambda_{k}=\Sigma \mu_{j}=1$. Hence

$$
x y=\sum_{j, k}\left(\lambda_{k} \mu_{j}\right)\left(e_{k} f_{j}\right)
$$

with $e_{k} f_{j} \in E, \lambda_{k} \mu_{j} \geqslant 0$ and

$$
\sum_{j, k} \lambda_{k} \mu_{j}=\left(\sum_{k} \lambda_{k}\right)\left(\sum_{j} \mu_{j}\right)=1 .
$$

Therefore $x y \in \operatorname{co}(E)$. Now suppose that $x \in \overline{\operatorname{co}}(E)$ and $y \in \operatorname{co}(E)$. Then there exists a net $\left\{x_{\alpha}\right\}$ in $\operatorname{co}(E)$ such that $\lim x_{\alpha}=x$. Since $x_{\alpha} y \in \operatorname{co}(E)$ for all $\alpha$, we have $x y=\lim x_{\alpha} y \in \overline{\operatorname{co}}(E)$. Finally, suppose that both $x, y$ are in $\overline{\operatorname{co}}(E)$. Then there is a net $\left\{y_{\alpha}\right\}$ in $\operatorname{co}(E)$ such that $\lim y_{\alpha}=y$. Since $x y_{\alpha} \in \overline{\operatorname{co}}(E)$ for all $\alpha$, we have $x y=\lim x y_{\alpha} \in \overline{\operatorname{co}}(E)$. Thus we have shown that $J$ is closed under multiplication. Replace $J$ by $1-J$ and $E$ by $1-E$, it follows that $1-J$ is also closed under multiplication. Therefore $J$ is a spectral carrier.

From the above proposition, we see that, if $E$ is a lattice of commuting idempotents contained in a compact convex set in $A$, then $E$ is contained in a complete lattice of commuting idempotents in $A$. From the same proposition, we see that, if $E$ is a sublattice of $\partial_{e} K$, where $K$ is a spectral carrier, then $\overline{c o}(E)$ is a spectral carrier contained in $K$. It is not hard to see that, conversely, every spectral carrier contained in $K$ is of the form $\overline{c o}(E)$, where $E$ is a sublattice of $\partial_{e} K$.

For the rest of this section, we consider some examples and applications to operators defined on a Hilbert space $H$.

For real numbers $\alpha, \beta$ with $\alpha<\beta$, we write $\mathscr{G} \mathscr{P}[\alpha, \beta]$ for the set of polynomials with real coefficients such that $p(\alpha)=0, p(\beta)=1$ and $p$ is increasing on $[\alpha, \beta]$. It follows from the spectral theory for hermitian operators that, if $h$ is a hermitian operator on $H$ with its spectrum $\sigma(H)$ contained in $[\alpha, \beta]$, then, for each $p$ in $\mathscr{P} \mathcal{P}[\alpha, \beta]$, we have $\|p(h)\| \leqslant 1$. The converse also holds and thus we have a characterization of hermitian operators as follows:

Proposition 2.13. An operator $h$ on a Hilbert space $H$ is a hermitian operator with $\sigma(h) \subseteq[\alpha, \beta]$ if and only if for all $p \in \mathscr{S} \mathscr{P}[\alpha, \beta],\|p(h)\|<1$.

Proof. To show the "if" part, let $K$ be the closure of $\{p(h): p \in \mathscr{G} \mathscr{P}[\alpha, \beta]\}$ in the weak operator topology. Since both $\mathscr{G} \mathscr{P}[\alpha, \beta]$ and $1-\mathscr{G} \mathscr{P}[\alpha, \beta]$ are convex and closed under multiplication and $K$ is contained in the unit ball of $B(H)$ which 
is compact in the weak operator topology, $K$ is a spectral carrier. Suppose that $e \in \partial_{e} K$. Then, by Proposition $2.2, e^{2}=e$. Since we also have $\|e\|<1, e$ must be a projection. In particular, $e$ is hermitian. By Kreĭn-Mil'man's Theorem, all elements in $K$ are hermitian. Let $p_{0}$ be the polynomial defined by

$$
p_{0}(x)=(\beta-\alpha)^{-1}(x-\alpha) \text {. }
$$

Then $p_{0} \in \mathscr{G} \mathscr{P}[\alpha, \beta]$. Thus $p_{0}(h)=(\beta-\alpha)^{-1}(h-\alpha) \in K$ and hence $h$ is hermitian. Since $K$ is the closed convex hull of a set of projections, every element $k$ in $K$ satisfies $0 \leqslant k \leqslant 1$. In particular, $0 \leqslant(\beta-\alpha)^{-1}(h-\alpha) \leqslant 1$ from which it follows $\alpha \leqslant h \leqslant \beta$, or $\sigma(h) \subseteq[\alpha, \beta]$.

REMARK. If the condition $\|p(h)\| \leqslant 1$ for all $p$ in $\mathscr{G} \mathscr{P}[\alpha, \beta]$ is replaced by the weaker condition that there exists a positive number $M$ such that $\|p(h)\|<M$ for all $p$ in $\mathscr{G} \mathscr{P}[\alpha, \beta]$, then $K$, the closure of $\{p(h): p \in \mathscr{P}[\alpha, \beta]\}$, is still a spectral carrier. If $h$ is a well-bounded operator on $H$ according to Smart [9], that is, there exist constants $\alpha, \beta, M$ with $\alpha<\beta$ and $M>0$ such that

$$
\|p(h)\| \leqslant M(|p(\alpha)|+\text { total variation of } p \text { over }[\alpha, \beta])
$$

for every polynomial $p$, then

$$
\|p(h)\| \leqslant M \text { if } p \in \mathscr{P}[\alpha, \beta]
$$

and hence $(\beta-\alpha)^{-1}(h-\alpha)$ is contained in a spectral carrier.

Now we give an alternative proof of Theorem XVII.2.5 in Dunford and Schwartz [4] in case that the underlying space is a Hilbert space.

Proposition 2.14. Let $A$ be an algebra in $B(H)$ which is the image under $a$ continuous homomorphism $\phi$ of the algebra $C(\Lambda)$ of all complex continuous functions on a compact space $\Lambda$. Then there exists an invertible element $s$ in $B(H)$ such that $s^{-1} A s=\left\{s^{-1}\right.$ as: $\left.a \in A\right\}$ is a commutative $C^{*}$-algebra of normal operators.

Proof. By assumption, there exists a positive number $M$ such that $\|\phi(f)\|<$ $M\|f\|_{\infty}$ for all $f \in C(\Lambda)$. Let $K$ be the closure of $\{\phi(f): f \in C(\Lambda), 0<f<1\}$ in the weak operator topology. Then it is easy to check that $K$ is a spectral carrier with $K=1-K$. Let $E$ be the set of all idempotents in $K$. Then it is easy to see that $E$ is a bounded Boolean algebra of idempotents. By [4, Lemma XV.6.2], there is an invertible operator $s$ such that $s^{-1} E s$ consists of projections. By Proposition 2.2, $\partial_{e} K \subseteq E$ and hence, by Krein-Mil'man's Theorem, $s^{-1} K s$ consists of hermitian operators. Since every element in $s^{-1} A s$ is a linear combination of elements in $s^{-1} K s, s^{-1} A s$ consists of commuting normal operators. Now it is clear that the mapping $\psi: C(\Lambda) \rightarrow B(H)$ given by $\psi(f)=s^{-1} \phi(f) s$ is a homomorphism from $C(\Lambda)$ into a commutative $C^{*}$-algebra. Hence $\psi$ must be ${ }^{*}$-preserving and $s^{-1} A s$, the image of $\psi$, must be a $C^{*}$-algebra.

3. Simplex and chain. If $K$ is a metrizable carrier in a locally convex algebra $A$, then, by Choquet's theory, $\partial_{e} K$ is a $G_{\delta}$-set and, for each $x \in K$, there exists a probability measure $\mu$ on $K$ such that $\mu\left(\partial_{e} K\right)=1$ and

$$
x=\int_{\partial_{e} K} e d \mu(e) .
$$


The last identity means that, for every continuous linear functional $\phi$,

$$
\phi(x)=\int_{\partial_{e} K} \phi(e) d \mu(e) .
$$

The compact convex set $K$ is said to be a simplex if, for each $x$, the measure $\mu$ described as above is uniquely determined by $x$. For the case when $K$ is not necessarily metrizable, the above statements have appropriate generalizations. For details, see [1], [2].

By Proposition 1.1, the spectral carrier $K$ is a simplex if the lattice $\partial_{e} K$ is finite and totally ordered. The main result of the present section is: a metrizable spectral carrier is a simplex if and only if the lattice $\partial_{e} K$ is totally ordered. The "only if" part is straightforward to prove

Proposition 3.1. If a spectral carrier $K$ is a simplex, then the lattice $\partial_{e} K$ of idempotents is totally ordered.

Proof. Let $e_{1}, e_{2} \in K$. Then $e_{1} e_{2}$ and $e_{1}+e_{2}-e_{1} e_{2}$ are in $\partial_{e} K$. Since

$$
\frac{1}{2}\left(e_{1}+e_{2}\right)=\frac{1}{2}\left(e_{1} e_{2}+\left(e_{1}+e_{2}-e_{1} e_{2}\right)\right),
$$

by the assumption that $K$ is a simplex, we have either $e_{1}=e_{1} e_{2}$ or $e_{2}=e_{1} e_{2}$.

For convenience, we introduce the following definition.

Definition 3.1. A set $C$ of commuting idempotents in an algebra is said to be a chain, if, for all $e_{1}, e_{2}$ in $C$, either $e_{1} \leqslant e_{2}$ or $e_{2} \leqslant e_{1}$.

Proposition 3.2. If $K$ is a spectral carrier and $\partial_{e} K$ is totally ordered, then $\partial_{e} K$ is closed and the multiplication in $\partial_{e} K$ is jointly continuous.

Proof. Let $\left\{e_{\alpha}: \alpha \in D\right\}$ be a convergent net of idempotents in $K$ and $x=$ $\lim e_{\alpha}$. We claim that $\left\{e_{\alpha}\right\}$ has a monotone subnet. In fact, if there exists some $\alpha_{0} \in D$ such that $\left\{e_{\alpha}: \alpha \geqslant \alpha_{0}\right\}$ is decreasing, then we are done. Otherwise, for each $\alpha_{1} \in D$, there exists some $\alpha_{2} \in D$ such that $e_{\alpha_{2}} \geqslant e_{\alpha_{1}}$ and, by means of Zorn's lemma, we can choose an increasing subnet from $\left\{e_{\alpha}\right\}$. By Theorem 2.11(c), every monotone net in $\partial_{e} K$ converges to an idempotent. Hence $x \in \partial_{e} K$. This shows that $\partial_{e} K$ is closed. By using a similar argument, we can show the ordering $<$ in $\partial_{e} K$ is closed, that is, $\left\{(e, f): e, f \in \partial_{e} K, e \leqslant f\right\}$ is a closed subset of $\partial_{e} K \times \partial_{e} K$. Now the second part of the proposition follows from the following lemma.

LEMMA 3.3. Let $K$ be a spectral carrier. Suppose that $\partial_{e} K$ is closed and the ordering $<$ in $\partial_{e} K$ is closed, then the multiplication in $\partial_{e} K$ is jointly continuous.

Proof. Since $\partial_{e} K$ is compact, it suffices to show that if $\left\{e_{\alpha}\right\}$ and $\left\{f_{\alpha}\right\}$ are nets with the same directed set, $\lim e_{\alpha}=e, \lim f_{\alpha}=f$ and $\lim e_{\alpha} f_{\alpha}=g$, then $e f=g$. From the fact that $e_{\alpha} f_{\alpha} \leqslant e_{\alpha}$ and the assumption that $\leqslant$ is closed, we have

$$
g=\lim e_{\alpha} f_{\alpha} \leqslant \lim e_{\alpha}=e .
$$

Similarly, we have $g \leqslant f$. Hence $g \leqslant e f$. On the other hand, since $\partial_{e} K$ is closed and

$$
e_{\alpha} \vee f_{\alpha}=e_{\alpha}+f_{\alpha}-e_{\alpha} f_{\alpha} \in \partial_{e} K
$$


for all $\alpha, e+f-g=\lim e_{\alpha} \vee f_{\alpha} \in \partial_{e} K$. Hence the element $e+f-g$ is an idempotent. Therefore

$$
\begin{aligned}
e+f-g & =(e+f-g)^{2}=e+f+g+2 e f-2 e g-2 f g \\
& =e+f+g+2 e f-4 g
\end{aligned}
$$

from which we obtain $g=e f$.

COROLlary 3.4. If $C$ is a chain of idempotents in a locally convex algebra and if $K=\overline{\operatorname{co}}(C)$ is compact, then $K$ is a spectral carrier and $\partial_{e} K$ is a chain of idempotents containing $C$ as a dense subchain.

Proof. By Proposition 2.12, $\mathrm{K}$ is a spectral carrier. By the proof of Proposition 3.2, we can show that $\bar{C}$, the closure of $C$, is a chain of idempotents. By a well-known result (e.g. [3, V.8.5]), we have $\partial_{e} K \subseteq \bar{C}$. On the other hand, by Theorem 2.10, $\bar{C} \subseteq \partial_{e} K$. Hence we have $\partial_{e} K=\bar{C}$.

The proof of the main result of the present section, which is the converse of Proposition 3.2 (under the extra assumption that $K$ is metrizable), is divided into two stages. First we prove a special case: if $S$ is a spectral carrier in $B(H)$ with $\partial_{e} S$ forming a chain of projections, then $S$ is a simplex. Secondly, we treat the general case by establishing a "covering simplex" $S$ in $B(H)$ and showing that the "covering map" is an "isomorphism" between $S$ and $K$.

Now, let $h$ be a hermitian operator defined on a Hilbert space $H$ with the property that $0 \leqslant h \leqslant 1$. Let $h=\int_{0}^{1} \lambda d e_{\lambda}$ be its spectral decomposition, where $\left\{e_{\lambda}\right\}$ is a resolution of unity which is continuous from the right. Let $C$ be the closure of the chain $\left\{1-e_{\lambda}: 0 \leqslant \lambda \leqslant 1\right\}$. Then it follows from Corollary 3.4 that all elements in $C$ are idempotents. On the other hand, $\|e\|<1$ for all $e$ in $C$. Therefore $C$ is a chain of projections. Let $\mu$ be the measure defined on $C$ by assigning $\mu(A)$ to be the Lebesgue measure of the set $\left\{\lambda: 0 \leqslant \lambda \leqslant 1,1-e_{\lambda} \in A\right\}$ for every Borel set $A$ in $C$. Then, for $\xi \in H$, we have

$$
\begin{aligned}
\langle h \xi, \xi\rangle & =\int_{0}^{1} \lambda d\left\langle e_{\lambda} \xi, \xi\right\rangle=\left.\lambda\left\langle e_{\lambda} \xi, \xi\right\rangle\right|_{0} ^{1}-\int_{0}^{1}\left\langle e_{\lambda} \xi, \xi\right\rangle d \lambda \\
& =\int_{0}^{1}\left\langle\left(1-e_{\lambda}\right) \xi, \xi\right\rangle d \lambda=\int_{C}\langle e \xi, \xi\rangle d \mu(e) .
\end{aligned}
$$

Since linear functionals of the form $x \rightarrow\langle x \xi, \xi\rangle$ with $\xi \in H$ separate points of $B(H)$, we have

$$
h=\int_{C} e d \mu(e)
$$

Thus we have shown that $h$ is the barycenter of a probability measure supported by a closed chain of projections, an expression obtained from the spectral decomposition of $h$ by means of integration by parts. Conversely, assuming that $h=$ $\int_{C} e d \mu(e)$, where $C$ is a closed chain of projections and $\mu$ is a probability measure supported by $C$, it is considerably more difficult to recover the resolution of identity $\left\{e_{\lambda}\right\}$ from $C$ and $\mu$ directly such that $h=\int_{0}^{1} \lambda d e_{\lambda}$; otherwise, we would 
prove the uniqueness of $\mu$ by means of the uniqueness of the spectral decomposition of $h$, and thus would show that the closed convex hull of $C$ is a simplex. The last statement, laid out as a theorem as follows, is proved in an indirect way.

THEOREM 3.5. If $C$ is a closed chain of projections in $B(H)$, where $H$ is a separable Hilbert space, then the closed convex hull of $C$ (in the weak operator topology) is a simplex.

Proof. Step I. We assume here that $C$ contains 0,1 and has no gap. (By a gap in $C$ we mean a pair $\left(e_{1}, e_{2}\right)$ of elements in $C$ with $e_{1} \leqslant e_{2}$ and $e_{1} \neq e_{2}$ such that, for all $e \in C$, either $e \leqslant e_{1}$ or $e \geqslant e_{2}$. See [5, Chapter I].)

Let $\left\{\xi_{n}\right\}$ be an orthonormal basis of $H$. Then the mapping $\phi: C \rightarrow[0,1]$ defined by

$$
\phi(e)=\sum_{n=1}^{\infty} 2^{-n}\left(e \xi_{n}, \xi_{n}\right)
$$

is one-one, continuous and order-preserving. Since $C$ has no gap, $C$ is connected and hence $\phi$ must be surjective. Now suppose that $\mu_{1}, \mu_{2}$ are probability measures supported by $C$ and

$$
h=\int_{C} e d \mu_{1}(e)=\int_{C} e d \mu_{2}(e) .
$$

Let $\nu_{j}=\mu_{j} \circ \phi^{-1}, e_{\lambda}=\phi^{-1}(\lambda)$ and $f_{j}(\lambda)=\nu_{j}[0, \lambda]$ for $j=1,2$ and $0<\lambda<1$. Then, for $\xi \in H$,

$$
\begin{aligned}
\langle h \xi, \xi\rangle & =\int_{C}\langle e \xi, \xi\rangle d \mu_{j}(e)=\int_{0}^{1}\left\langle e_{\lambda} \xi, \xi\right\rangle d \nu_{j}(\lambda) \\
& =\left.f_{j}(\lambda)\left\langle e_{\lambda} \xi, \xi\right\rangle\right|_{0} ^{1}-\int_{0}^{1} f_{j}(\lambda) d\left\langle e_{\lambda} \xi, \xi\right\rangle \\
& =\|\xi\|^{2}-\left\langle\left(\int_{0}^{1} f_{j}(\lambda) d e_{\lambda}\right) \xi, \xi\right\rangle .
\end{aligned}
$$

Hence we have $\int_{0}^{1} f_{1}(\lambda) d e_{\lambda}=\int_{0}^{1} f_{2}(\lambda) d e_{\lambda}$. Since both $f_{1}$ and $f_{2}$ are nondecreasing, continuous from the right and the map $\lambda \rightarrow e_{\lambda}$ is continuous, strictly increasing, it is easy to check that $f_{1}=f_{2}$. Therefore $\nu_{1}=\nu_{2}$ which in turn implies $\mu_{1}=\mu_{2}$.

Step II. Now we consider the general case. Let $\phi: B(H) \rightarrow B(H \otimes H)$ be the mapping defined by $\phi(x)=x \otimes 1$. Then $\phi(C)$ is closed chain of projections in $B(H \otimes H)$. It is easy to check that, for $e_{1}, e_{2} \in C$, the pair $\left(e_{1}, e_{2}\right)$ is a gap in $C$ if and only if $\left(\phi\left(e_{1}\right), \phi\left(e_{2}\right)\right)$ is a gap in $\phi(C)$ and, in such case, the rank of the projection $\phi\left(e_{2}\right)-\phi\left(e_{1}\right)=+\infty$. Hence there is a closed chain $\tilde{C}$ of projections in $B(H \otimes H)$ such that $\phi(C) \subseteq \tilde{C}$ and $\tilde{C}$ has no gap. (For details, see [5, pp. 17-18].) Now suppose that

$$
h=\int_{C} e d \mu_{j}(e) \quad(j=1,2) .
$$

Let $\nu_{j}=\mu_{j}^{\circ} \phi^{-1}$. Then $\nu_{j}$ is a measure on $\phi(C)$ and hence can be regarded as a measure on $\tilde{C}$. We have

$$
h \otimes 1=\int_{C}(e \otimes 1) d \mu_{j}(e)=\int_{\tilde{C}} f d v_{j}(f)
$$


By Step I, we have $\nu_{1}=\nu_{2}$. Since $\phi$ is a homeomorphism between $C$ and $\phi(C)$, we must have $\mu_{1}=\mu_{2}$.

Proposition 3.6. Let $C$ be a chain of projections in $B(H)$ and $K=\overline{\operatorname{co}}(C)$, where the Hilbert space $H$ is not necessarily separable. Then the strong operator topology in $K$ coincides with the weak operator topology.

Proof. From a topological consideration, we see that it suffices to show that $K$ is compact under the strong operator topology. Since the unit ball of $B(H)$ is complete in the strong operator topology, by a well-known fact concerning compact sets in a topological vector space (e.g., see [8, p. 50, Corollary II 4.3]) it suffices to show that $C$ is compact in the strong operator topology. Now, if $\left\{e_{\nu}\right\}$ is a net in $C$, then, by an argument used in the proof of Proposition $3.4,\left\{e_{\nu}\right\}$ has a monotone subnet. It is well known that every monotone net of projections on $H$ is strongly convergent. Therefore $C$ is compact.

Now we return to the general theory. For the rest of this section, we always assume that $K$ is a metrizable spectral carrier with $\partial_{e} K$ totally ordered. For technical reasons, we also assume that $K$ contains 0 and 1 . Our goal is to show that $K$ is a simplex.

LEMMA 3.7. There is an order-preserving homeomorphism $\psi$ from $\partial_{e} K$ onto some compact set $M$ in $[0,1]$ such that $\psi(0)=0$ and $\psi(1)=1$.

Proof. By modifying the proof of Urysohn's lemma, for given $e_{1}, e_{2} \in \partial_{e} K$ with $e_{1} \leqslant e_{2}, e_{1} \neq e_{2}$, one can construct a continuous increasing function $\phi: \partial_{e} K \rightarrow[0,1]$ such that $\phi\left(e_{1}\right)=0$ and $\phi\left(e_{2}\right)=1$. (For details, see [6, Theorem 1.2.1].) It suffices to show that there exists a sequence $\left\{\psi_{n}\right\}$ of continuous increasing functions from $\partial_{e} K$ into $[0,1]$ which separates points of $\partial_{e} K$ with $\psi_{n}(0)=0$ and $\psi_{n}(1)=1$; for then we can set

$$
\psi=\sum_{n=1}^{\infty} 2^{-n} \psi_{n}
$$

which is a function having the required properties. To this end, first we show that $\partial_{e} K$ has at most countably many gaps. Let $\rho$ be a metric on $K$. For each positive integer $k$, let $G_{k}$ be the collection of all gaps $(e, f)$ with $\rho(e, f)>k^{-1}$. Then $G_{k}$ is a finite collection. Otherwise, by an argument used in Proposition 3.2, we can show that there exists a sequence $\left(e_{n}, f_{n}\right)$ in $G_{k}$ such that $\left\{e_{n}\right\}$ is strictly monotone. For definiteness, we assume that $\left\{e_{n}\right\}$ is strictly increasing. We have

$$
e_{1} \leqslant f_{1} \leqslant e_{2} \leqslant f_{2} \leqslant e_{3} \leqslant f_{3} \leqslant \ldots
$$

By Theorem 2.11(c), both $\left\{e_{n}\right\}$ and $\left\{f_{n}\right\}$ are convergent to the same limit. But, on the other hand,

$$
\rho\left(\lim e_{n}, \lim f_{n}\right)=\lim \rho\left(e_{n}, f_{n}\right)>k^{-1} .
$$

Thus we arrive at a contradiction. Now it is clear that the collection of all gaps, namely, $\cup_{k} G_{k}$, is at most countable. Let $D$ be a countable dense subset of $\partial_{e} K$. Let $P$ be the collection of all those pairs $(e, f)$ with $e, f \in C, e<f, e \neq f$, such that either $(e, f)$ is a gap of $C$ or both $e, f$ are in $D$. Then $D$ is countable and hence can 
be arranged into a sequence, say, $D=\left\{\left(e_{n}, f_{n}\right): n=1,2, \ldots\right\}$. For each $n$, let $\psi_{n}$ be a continuous increasing function on $\partial_{e} K$ into $[0,1]$ such that $\psi_{n}\left(e_{n}\right)=0$ and $\psi_{n}\left(f_{n}\right)=1$. It is not hard to see that $\left\{\psi_{n}\right\}$ separates points of $\partial_{e} K$.

LEMMA 3.8. There exists a closed chain $C$ of projections on a separable Hilbert space $H$ and a homeomorphism $\phi: C \rightarrow \partial_{e} K$ such that $\phi(0)=0, \phi(1)=1$ and $\phi\left(p_{1} p_{2}\right)=\phi\left(p_{1}\right) \phi\left(p_{2}\right)$ for all $p_{1}, p_{2} \in C$.

Proof. Let $\psi, M$ be the same as those in the previous lemma. Let $H=L^{2}[0,1]$. For $\lambda \in M$, let $p_{\lambda}$ be the projection sending $\xi \in L^{2}[0,1]$ to $\chi_{[0, \lambda]} \xi$. (Here $\chi_{[0, \lambda]}$ stands for the characteristic function of the closed interval $[0, \lambda]$.) Then $\lambda \rightarrow p_{\lambda}$ is a one-one, continuous and increasing mapping from $M$ onto a chain $C$ of projections. It is straightforward to check that the inverse mapping of $e \rightarrow p_{\psi(e)}$, where $e \in \partial_{e} K$, is the required mapping $\phi$. (Note that the condition $\phi\left(p_{1} p_{2}\right)=\phi\left(p_{1}\right) \phi\left(p_{2}\right)$ for all $p_{1}, p_{2}$ means the same as that $\phi$ is increasing.)

Let $C$ and $\phi$ be those described in Lemma 3.8. Let $S$ be the closure of $\operatorname{co}(C)$ in the weak operator topology. By Theorem 3.5, $S$ is a simplex. Hence, for each $x \in S$, there exists a unique probability measure $\mu_{x}$ on $C$ such that $x=\int_{C} p d \mu_{x}(p)$. Define $\tilde{\phi}: S \rightarrow K$ by putting

$$
\tilde{\phi}(x)=\int_{\partial_{e} K} e d\left(\mu_{x} \circ \phi^{-1}\right)(e) .
$$

It is not hard to check that $\tilde{\phi}$ is continuous and affine. (For details, see [1, Theorem II 4. 1].) Note that if $x \in \operatorname{co}(C)$, say, $x=\sum \lambda_{k} p_{k}$ with $\lambda_{k}>0$ and $\sum \lambda_{k}=1$, then $\tilde{\phi}(x)=\sum \lambda_{k} \phi\left(p_{k}\right)$. From the fact that $\phi(p q)=\phi(p) \phi(q)$ for all $p, q \in C$, it is easy to check that, if $x, y \in \operatorname{co}(C)$, then $\tilde{\phi}(x) \tilde{\phi}(y)=\tilde{\phi}(x y)$. By the continuity of $\tilde{\phi}$ and the denseness of $\operatorname{co}(C)$ in $S$, the last identity holds for all $x, y \in S$. Thus we obtain

Proposition 3.9. There exists a continuous affine mapping $\phi$ from $S=\overline{\operatorname{co}}(C)$ onto $K$ such that for all $x, y \in S, \phi(x y)=\phi(x) \phi(y)$ and the restriction of $\phi$ to $C$ is one-one and onto $\partial_{e} K$.

COROLlary 3.10. The multiplication in $K$ is jointly continuous.

Proof. By the compactness of $K$, it suffices to show that if $a_{n}, b_{n} \in K$, $\lim b_{n}=b$ and $\lim a_{n} b_{n}=c$, then $c=a b$. Let $x_{n}, y_{n} \in S$ be such that $\phi\left(x_{n}\right)=a_{n}$ and $\phi\left(y_{n}\right)=b_{n}$. By taking a subsequence if necessary, we may assume that both $\left\{x_{n}\right\}$ and $\left\{y_{n}\right\}$ are convergent in the weak operator topology, say $x=\lim x_{n}$ and $y=\lim y_{n}$. By Proposition 3.6, the weak operator topology and the strong operator topology in $S$ coincide. Therefore we have $x y=\lim x_{n} y_{n}$. Now

$$
\begin{aligned}
a b & =\phi(x) \phi(y)=\phi(x y)=\lim \phi\left(x_{n} y_{n}\right) \\
& =\lim \phi\left(x_{n}\right) \phi\left(y_{n}\right)=\lim a_{n} b_{n}=c .
\end{aligned}
$$

Next we develop a functional calculus for elements in $K$. We denote by $G[0,1]$ the set of all real-valued functions $f$ defined on $[0,1]$ such that $f(0)=0, f(1)=1$ 
and $f$ is monotonely increasing on $[0,1]$. Recall that $\mathscr{P} \mathscr{P}[0,1]$ is the set of those functions in $\mathscr{G}[0,1]$ which are polynomials. We write $\mathscr{G}[0,1]$ for all those functions in $\mathscr{G}[0,1]$ which are continuous.

LeMmA 3.11. If $a \in K$ and $p \in \mathscr{G} \mathscr{P}[0,1]$, then $p(a) \in K$.

Proof. Using summation by parts (see the proof of $(a) \Rightarrow(b)$ in Proposition 1.1), we can show that an element $b$ in $K$ is a convex combination of $\partial_{e} K$ if and only if it can be expressed as $b=\sum_{j=1}^{n} \mu_{j} f_{j}$, where $0<\mu_{1}<\mu_{2}<\cdots<\mu_{n}<1, f_{j}$ are idempotents satisfying $f_{j} f_{k}=0$ if $j \neq k$ and $e_{k}=\sum_{j=k}^{n} f_{j} \in \partial_{e} K$ for $k=1, \ldots, n$. For such $b$, we have

$$
p(b)=\sum_{j=1}^{n} p\left(\mu_{j}\right) f_{j}
$$

with $0 \leqslant p\left(\mu_{1}\right)<p\left(\mu_{2}\right)<\cdots<p\left(\mu_{n}\right) \leqslant 1$. Thus the lemma holds for convex combination of $\partial_{e} K$. In general, we take a sequence $\left\{b_{n}\right\}$ of convex combinations of $\partial_{e} K$ such that $\lim b_{n}=a$. By Corollary 3.10 , we have $\lim p\left(b_{n}\right)=p(a)$. Since $p\left(b_{n}\right) \in K$ for each $n$, we have $p(a) \in K$.

LEMMA 3.12. (a) If $g \in \mathscr{G}[0,1]$, then there is a sequence $\left\{q_{n}\right\}$ in $\mathscr{S} \mathcal{P}[0,1]$ such that

$$
\left\|q_{n}-g\right\|_{\infty}=\sup _{0<\lambda<1}\left|q_{n}(\lambda)-g(\lambda)\right| \rightarrow 0 \text { as } n \rightarrow \infty .
$$

(b) If $f \in \mathscr{G}[0,1]$, then there exists a sequence $\left\{r_{n}\right\}$ in $\mathscr{P} \mathscr{G}[0,1]$ such that $\lim r_{n}(\lambda)=f(\lambda)$ for all $\lambda \in[0,1]$.

Proof. (a) By means of a smoothing process, it is not difficult to show that $g$ can be uniformly approximated by $C^{\infty}$-functions in $g[0,1]$. Since $g$ is increasing, $g^{\prime}(\lambda)>0$ for all $\lambda \in[0,1]$. Consider the Bernstein polynomials

$$
b_{n}(\lambda)=\sum_{k=0}^{n} g^{\prime}\left(\frac{k}{n}\right)\left(\begin{array}{l}
n \\
k
\end{array}\right) \lambda^{k}(1-\lambda)^{n-k} \quad(0<\lambda<1)
$$

and let $p_{n}(\lambda)=\int_{0}^{\lambda} b_{n}(\xi) d \xi$. Then $b_{n}(\lambda) \geqslant 0$ for all $\lambda$ and hence $p_{n}$ is a polynomial increasing on $[0,1]$. Since $\left\|b_{n}-g^{\prime}\right\|_{\infty} \rightarrow 0$, we have $\left\|p_{n}-g\right\|_{\infty} \rightarrow 0$. Let $q_{n}=$ $p_{n}(1)^{-1} p_{n}$. Then $q_{n}$ is the required sequence.

(b) Note that $f$ can be expressed as

$$
f=\varepsilon_{0} f_{0}+\sum_{n=1}^{\infty} \varepsilon_{n} \chi_{n}
$$

where $\varepsilon_{n} \geqslant 0$ for all $n \geqslant 0, \sum_{n=0}^{\infty} \varepsilon_{n}=1, f_{0} \in \mathscr{S}[0,1]$ and, for each $n>1, \chi_{n}$ is a function in $\mathscr{G}[0,1]$ such that, for all $\lambda \in[0,1]$, possibly with one exceptional point, the value of $\chi_{n}(\lambda)$ is either 0 or 1 . It is not difficult to show that each $\chi_{n}$ is the pointwise limit of a sequence in $\mathscr{S}[0,1]$. Now (b) follows from (a). 
Proposition 3.13. For each $a \in K$, there is a mapping from $\mathscr{G}[0,1]$ into $K$, denoted by $f \rightarrow f(a)$, such that:

(a) If $p \in \mathscr{P}[0,1]$, then $p(a)$ has the usual meaning.

(b) For $f, g \in \mathcal{G}[0,1]$ and $0 \leqslant \lambda \leqslant 1$, we have

$$
\begin{aligned}
(f g)(a) & =f(a) g(a), \\
(f \circ g)(a) & =f(g(a)), \\
(\lambda f+(1-\lambda) g)(a) & =\lambda f(a)+(1-\lambda) g(a) .
\end{aligned}
$$

(c) If $f_{n} \in \mathscr{G}[0,1]$ and $f_{n} \rightarrow f$ pointwisely, then $f_{n}(a) \rightarrow f(a)$.

Proof. Take any $x \in S$ such that $\phi(x)=a$. (The symbols $S$ and $\phi$ are the same as those in Proposition 3.9.) For $f \in \mathscr{G}[0,1]$, let $f(a)$ be defined by putting $f(a)=\phi(f(x))$. We have to show that $f(a)$ does not depend on the choice of $x$. By Lemma 3.12(b), there exists a sequence $\left\{p_{n}\right\}$ in $\mathscr{S} \mathscr{P}[0,1]$ such that $\lim p_{n}(\lambda)=f(\lambda)$ for all $\lambda \in[0,1]$. By Lebesgue's dominated convergence theorem, one can show that $p_{n}(x)$ converges to $f(x)$ in the strong operator topology. Hence

$$
\phi(f(x))=\phi\left(\lim p_{n}(x)\right)=\lim \phi\left(p_{n}(x)\right)=\lim p_{n}(a) .
$$

The $\operatorname{limit} \lim p_{n}(a)$ is certainly independent of the choice of $x$. Hence the expression $f(a)$ is well defined. The rest of the proof is routine and hence omitted.

Now we can state and prove the main theorem of the present section.

THEOREM 3.14. If $K$ is a metrizable spectral carrier and if $\partial_{e} K$ is a chain of idempotents, then $K$ is a simplex.

Proof. Let $\phi: S \rightarrow K$ be the affine mapping constructed in Proposition 3.9. Since, by Theorem 3.5, $S$ is a simplex, it suffices to show that $\phi$ is one-one. Let $x_{1}$, $x_{2} \in S$ be such that $\phi\left(x_{1}\right)=\phi\left(x_{2}\right)=a$. For $\lambda \in(0,1]$, define $f_{\lambda}$ by

$$
f_{\lambda}(\xi)= \begin{cases}0 & \text { if } \xi<\lambda, \\ 1 & \text { if } \xi \geqslant \lambda .\end{cases}
$$

Then, according to the proof of Proposition 3.13, $f_{\lambda}(a)=\phi\left(f_{\lambda}\left(x_{j}\right)\right), j=1,2$. Since $f_{\lambda}^{2}=f_{\lambda}, f_{\lambda}\left(x_{j}\right)$ is a projection and hence is in $C$. Since $\phi$ is one-one on $C$, we have $f_{\lambda}\left(x_{1}\right)=f_{\lambda}\left(x_{2}\right)$ for all $\lambda \in(0,1]$. Now, by the spectral theorem for hermitian operators, we have $x_{1}=x_{2}$. Hence $\phi$ is one-one and thus $K$ is a simplex.

A spectral carrier which is also a simplex is naturally called a simplicial spectral carrier, or simply called a simplicial carrier. Theorem 3.14 and Proposition 3.1 say that a metrizable spectral carrier is simplicial if and only if its extreme points form a chain of idempotents. From Choquet Theory's point of view, elements in a simplicial carrier are nice. A natural question is, when is an element contained in a simplicial carrier? In particular, if an element is contained in a spectral carrier, is it necessarily contained in a simplicial carrier? Here we only give a rather modest partial answer to this question.

Proposition 3.15. If $K$ is a metrizable spectral carrier such that (a) $\partial_{e} K$ is closed and (b) the ordering $\leqslant$ in $\partial_{e} K$ is closed, then each element in $K$ is contained in a simplicial carrier. 
Proof. Let $\rho$ be a metric in $K$ and let $x \in K$. By Kreĭn-Mil'man's Theorem, there is a sequence $\left\{x_{n}\right\}$ in $\operatorname{co}\left(\partial_{e} K\right)$ such that $\lim \rho\left(x, x_{n}\right)=0$. For each $n, x_{n}$ is a convex combination of commuting idempotents and hence, by Proposition 1.1, $x_{n}$ is contained in a finite-dimensional simplex, say, $\operatorname{co}\left(C_{n}\right)$, where $C_{n}$ is a finite chain of idempotents. Let $S_{n}=\operatorname{co}\left(C_{n}\right) \cap K$. It is straightforward to check that $S_{n}$ is a spectral carrier. Since an element is an idempotent in $S_{n}$ if and only if it is an idempotent in both $\operatorname{co}\left(C_{n}\right)$ and $K$, we have $\partial_{e} S_{n}=C_{n} \cap \partial_{e} K$. Therefore $\partial_{e} S_{n}$ is a chain and thus $S_{n}$ is a simplex. Without the loss of generality, we may assume $\operatorname{co}\left(C_{n}\right)=S_{n}$ and thus $x_{n} \in S_{n}$ and $\partial_{e} S_{n}=C_{n} \subseteq \partial_{e} K$. Recall that lim $\sup _{n} C_{n}$ (resp. $\lim \inf _{n} C_{n}$ ) is the set of all those elements $y$ in $K$ such that, for every neighborhood $V_{y}$ of $y, V_{y} \cap C_{n} \neq \varnothing$ for infinitely many $n$ (resp., for all except finitely many $n$ ). By a well-known result in general topology (see, e.g., [10, Theorem I.7.1]), $\left\{C_{n}\right\}$ has a subsequence $\left\{C_{n_{k}}\right\}$ such that

$$
\underset{k}{\lim \sup } C_{n_{k}}=\liminf _{k} C_{n_{k}}(=C, \text { say }) .
$$

From $\lim \sup _{k} C_{n_{k}}=C$ and the compactness of $C$, we see that, for a given $\varepsilon>0$, there exists some $k_{0}$ such that, for $k \geqslant k_{0}$, we have

$$
C_{n_{k}} \subseteq\{y: \rho(y, C) \leqslant \varepsilon\}
$$

from which we obtain

$$
\operatorname{co}\left(C_{n_{k}}\right) \subseteq\{y: \rho(y, \overline{\operatorname{co}}(C)) \leqslant \varepsilon\} .
$$

Since $x_{n_{k}} \in \operatorname{co}\left(C_{n_{k}}\right)$ for all $k$ and $\rho\left(x_{n_{k}}, x\right) \rightarrow 0$ as $k \rightarrow \infty$, we have $\rho(x, \overline{\operatorname{co}}(C))<\varepsilon$. Since $\varepsilon>0$ is arbitrary, we have $x \in \operatorname{co}(C)$. It remains to show that $C$ is a chain of idempotents.

By assumption (a), we have $C \subseteq \partial_{e} K$. Let $e_{1}, e_{2} \in C$ with $e_{1} \neq e_{2}$. Since lim $\inf _{k} C_{n_{k}}=C$, there exist sequences $\left\{p_{k}\right\},\left\{q_{k}\right\}$ such that $p_{k}, q_{k} \in C_{n_{k}}$ and $\lim p_{k}=$ $e_{1}, \lim q_{k}=e_{2}$. For each $k$, we have either $p_{k} \leqslant q_{k}$ or $p_{k}>q_{k}$. Hence we have either $p_{k} \leqslant q_{k}$ for infinitely many $k$ or $p_{k} \geqslant q_{k}$ for infinitely many $k$. By assumption (b), we have either $e_{1} \geqslant e_{2}$ or $e_{1} \leqslant e_{2}$. Therefore $C$ is a chain.

COROLlARY 3.16. If $K$ is a metrizable spectral carrier in which the multiplication is jointly continuous, then each element in $K$ is contained in a simplicial carrier.

Remark. Let $x$ be an element in a locally convex algebra $A$. Suppose that $x$ is contained in a simplicial carrier. Then, by Lemma 3.11, the set

$$
S_{x}=\overline{\operatorname{co}}\{p(x): p \in \mathscr{S} \mathscr{P}[0,1]\}
$$

is the smallest simplicial carrier containing $x$. Since $\mathscr{G}[0,1]$ is compact in the pointwise-convergence topology, by Proposition 3.13(c), we have

$$
S_{x}=\{f(x): f \in g[0,1]\} \text {. }
$$

We may call $S_{x}$ the support of $x$. It is easy to see that, if $x$ is contained in a simplicial carrier, $y \in A$ and $y x=x y$, then $y s=s y$ for every $s$ in the support of $x$. Thus we obtain a version of Fuglede's theorem for such $x$. 


\section{REFERENCES}

1. E. M. Alfsen, Compact convex sets and boundary integrals, Ergebnisse der Mathematik und ihrer Grenzgebiete, Band 57, Springer-Verlag, New York, 1971.

2. G. Choquet, Lectures on analysis. Vol. II, Benjamin, New York, 1969.

3. N. Dunford and J. T. Schwartz, Linear operators. Part I: General theory, Interscience, New York, 1958.

4. Linear operators. Part III: Spectral operators, Interscience, New York, 1962.

5. I. C. Gohberg and M. G. Krein, Theory and applications of Volterra operators in Hilbert space, Transl. Math. Monographs, vol. 24, Amer. Math. Soc., Providence, R. I., 1970.

6. L. Nachbin, Topology and order, Van Nostrand, Princeton, N. J., 1965.

7. J. R. Ringrose, On well-bounded operators, J. Austral. Math. Soc. Ser. A 1 (1960), 334-343.

8. H. H. Schaefer, Topological vector spaces, Macmillan, New York, 1966.

9. D. R. Smart, Conditionally convergent spectral expansions, J. Austral. Math. Soc. Ser. A 1 (1960), 319-333.

10. G. T. Whyburn, Topological analysis, Princeton Univ. Press, Princeton, N. J., 1964.

Department of Mathematics and Statistics, University of Guelph, Guelph, Ontario, Canada N1G 2W1

Current address: Department of Mathematics, University of Toronto, Toronto, Ontario, Canada M5S 1A1 